\title{
Population Models for Massive Globular Clusters
}

\author{
Young-Wook Lee, Seok-Joo Joo, Sang-Il Han, Chongsam Na, \\ Dongwook Lim and Dong-Goo Roh \\ Center for Galaxy Evolution Research and Department of Astronomy, Yonsei University, \\ Seoul 120-749, Korea \\ email: ywlee2@yonsei.ac.kr
}

\section{Stellar Models with Enhanced CNO Abundance}

Increasing number of massive globular clusters (GCs) in the Milky Way are now turned out to host multiple stellar populations having different heavy element abundances enriched by supernovae. Recent observations have further shown that $[\mathrm{CNO} / \mathrm{Fe}]$ is also enhanced in metal-rich subpopulations in most of these GCs, including $\omega$ Cen and M22 (Marino et al. 2011, 2012). In order to reflect this in our population modeling, we have expanded the parameter space of $Y^{2}$ isochrones and horizontal-branch (HB) evolutionary tracks to include the cases of normal and enhanced nitrogen abundances $([\mathrm{N} / \mathrm{Fe}]=$ $0.0,0.8$, and 1.6). The observed variations in the total CNO content were reproduced by interpolating these nitrogen enhanced stellar models. Our test simulations with varying $\mathrm{N}$ and $\mathrm{O}$ abundances show that, once the total $\mathrm{CNO}$ sum $([\mathrm{CNO} / \mathrm{Fe}])$ is held constant, both $\mathrm{N}$ and $\mathrm{O}$ have almost identical effects on the HR diagram (see Fig. 1).

\section{New Calcium Narrow-band Photometry and Low Resolution Spectroscopy}

On the observational side, we are performing new Calcium narrow-band photometry using the new Calcium filter we designed to avoid CN contamination. The Calcium filter available at CTIO, the one used in Lee, J.-W. et al. (2009) and Roh et al. (2011), was suspected to be affected by some CN contamination, and this was recently confirmed, upon our request, by C. Johnson, D. Hölck, and A. Kunder (2012, private communication). Fortunately, we are still detecting two distinct red giant branches (RGBs) from the new filter for M22 and NGC 1851, suggesting that they are indeed different in Ca abundance. To confirm this, we have performed low-resolution spectroscopy for the stars on the two RGBs in each of these GCs, and have detected more than 4 sigma differences in spectroscopic $h k$ index for both M22 and NGC 1851. The difference is about 0.2 dex in $[\mathrm{Ca} / \mathrm{H}]$. We have also detected more than 8 sigma differences in $\mathrm{CN}$ abundance, and therefore the second generation stars in these clusters are enhanced in both Ca and CN. For the NGC 288, however, the presence of two distinct RGBs (Roh et al. 2011) is now turned out to be due to the large difference in $\mathrm{CN}$ abundance, and the $\mathrm{Ca}$ abundance difference is not confirmed from both new Calcium photometry and the spectroscopy.

\section{Population Models}

In order to investigate the star formation histories of these peculiar GCs, we have constructed synthetic CMDs for $\omega$ Cen, M22, and NGC 1851 (see Joo \& Lee 2013 for details). As described above, our models are based on the updated versions of $Y^{2}$ isochrones and 


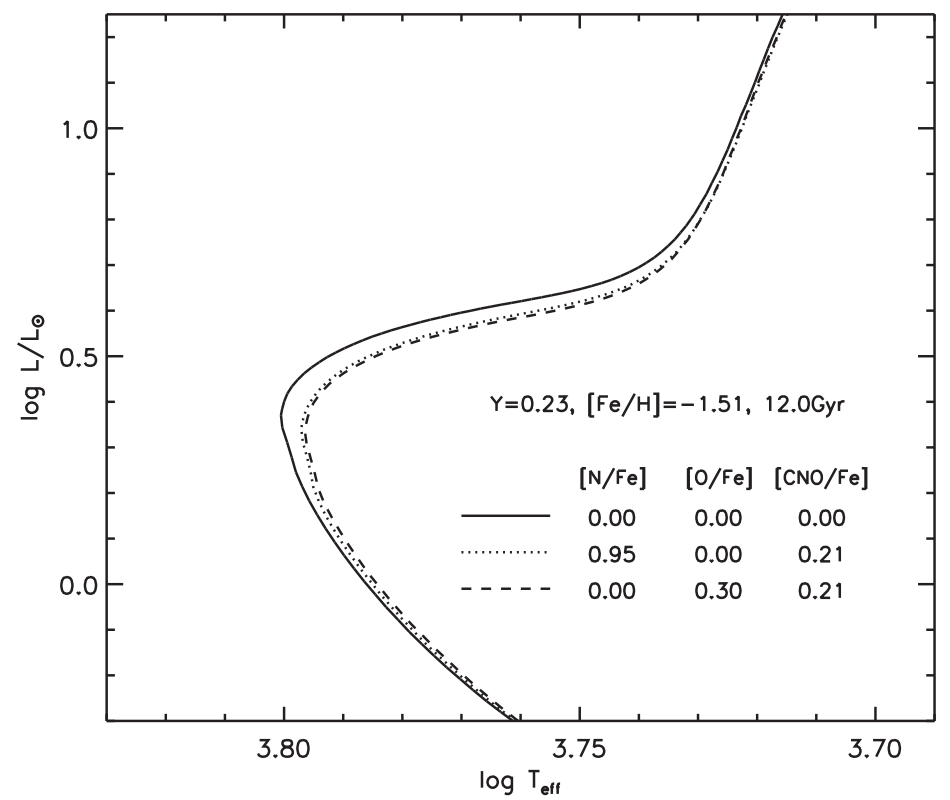

Figure 1. Two isochrones, one with enhanced N, and the other with enhanced O, compared to the reference case. In these two isochrones, $\mathrm{N}$ and $\mathrm{O}$ abundances are adjusted so that they all have the same value of $[\mathrm{CNO} / \mathrm{Fe}]$. Note that once the $\mathrm{CNO}$ sum is held constant, both $\mathrm{N} \& \mathrm{O}$ have almost identical effects on the HR diagram.

HB evolutionary tracks, with which the enhancements in both helium abundance and $[\mathrm{CNO} / \mathrm{Fe}]$ can be tested self-consistently. To estimate ages and helium abundances of subpopulations in each GC, we have compared our models with the observations (including our new Calcium photometry) on the Hess diagram by employing a Chi-square minimization technique. We find that metal-rich subpopulations in each of these GCs are also enhanced in helium abundance, and the age differences between the metal-rich and metal-poor subpopulations are fairly small (0.3 - 1.7 Gyr), even in the models with the observed variations in the total CNO content. These are required to simultaneously reproduce the observed extended $\mathrm{HB}$ and the splits on the main sequence, subgiant branch, and RGB. Our results are consistent with the hypothesis that these GCs are the relics of more massive primeval dwarf galaxies that merged and disrupted to form the proto-Galaxy.

\section{References}

Joo, S.-J \& Lee, Y.-W. 2013, ApJ, submitted

Lee, J.-W., Kang, Y.-W., Lee, J., \& Lee, Y.-W. 2009, Nature 462, 480

Marino, A. F., Sneden, C., Kraft, R. P., et al. 2011, A\&A 532, A8

Marino, A. F., Milone, A. P., Piotto, G., et al. 2012, ApJ 746, 14

Roh, D.-G., Lee, Y.-W., Joo, S.-J., Han, S.-I., Sohn, Y.-J., \& Lee, J.-W. 2011, ApJ 733, L45 